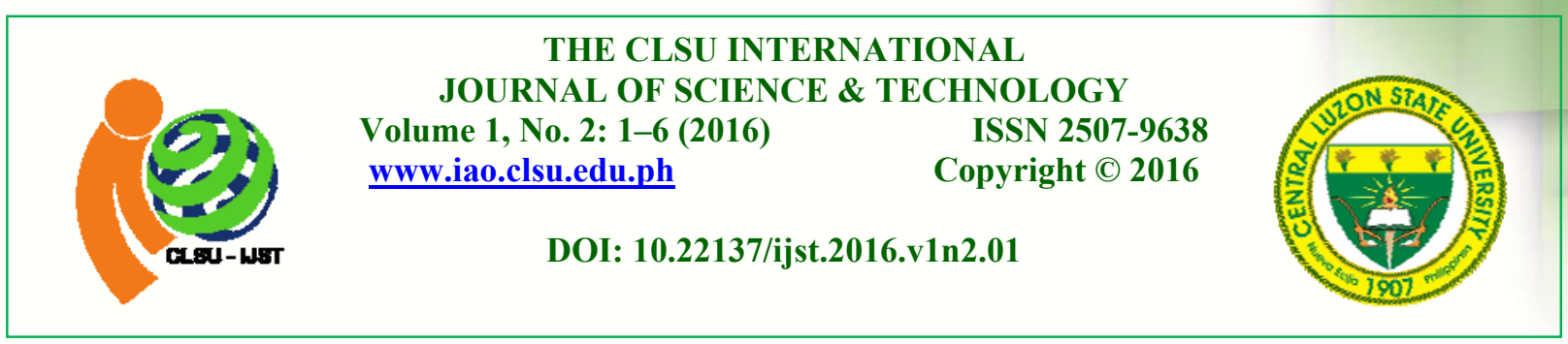

\title{
A Statistical Evaluation of In Vitro Micronucleus Assay in Toxicology
}

\author{
John Closter F. Olivo \\ Department of Statistics, College of Arts and Sciences, Central Luzon State University, Philippines \\ Email for correspondence: olivo.johncloster@yahoo.com \\ Submitted 25 April 2016, Accepted 08 September 2016, Published online 31 December 2016
}

\begin{abstract}
The study of DNA damage at the chromosome level is an essential part of genetic toxicology. Three different statistical aspects were considered in analyzing results from in vitro micronucleus assay. One aspect is concerned with the possible presence of overdispersion, the second aspect is the choice between p-values and confidence interval, and the third aspect is the type of inference. In this project, the possible genotoxic effect of $\mathrm{D} / \mathrm{L}$ Menthol was investigated by analyzing the frequencies of micronuclei in cultured lymphocytes exposed to D/L Menthol. Under the test conditions of the assay, D/L Menthol, either in the presence or absence of metabolic activation (S-9), did not induce a clear dose-dependent increase in the micronuclei occurrence. This result suggests that $\mathrm{D} / \mathrm{L}$ Menthol did not have a chromosomal-damaging effect in mammalian lymphocytes.
\end{abstract}

Keywords: D/L Menthol; in vitro; micronucleus; metabolic activation

\section{INTRODUCTION}

One of the most well established tests to screen new chemical entities with a widespread acceptance in industry is the in vitro micronucleus assay. As a measure for chromosomal aberrations in vitro, data on the frequency of micronucleus (MN) from a thousand of cells were statistically evaluated. The focus of this paper was restricted only to the statistical procedure for the analysis of micronuclei, particularly on the application of methodology as suggested by Hothorn and Gerhard (HG) (2008; 2009).

The predominant design for in vitro is a randomized one-way layout. A duplicate cell culture of mammalian origin is exposed to a series of test substance concentrations, i.e. a minimum of three dose levels, both with and without a source of metabolic activation. Concurrent vehicle and positive controls are included in all tests. During or after exposure to the test substance, the cells are grown for a period sufficient to allow chromosome damage to lead to the formation of micronuclei in interphase cells. Harvested and stained interphase cells are then analyzed for the 
presence of micronuclei (OECD, 2009). HG recommended taking into account the extra-variability since culture is the experimental unit.

The second statistical aspect is the contradiction between statistical significance and biological relevance. The use of p-values is the most commonly used in drawing out conclusions which represents a probability of falsification but do not provide interpretation in terms of biological relevance. For this case, HG recommended the use of confidence intervals over p-values.

The third aspect is the type of inference. HG dwelled upon the type of inference that is of relevance in trying to identify an increasing dose-related trend, possibly with downturn effects at high doses (Bretz and Hothorn 2002).

The aim of this project was to implement, apply, and discuss HG's recommendations using the genotoxicity database of $\mathrm{D} / \mathrm{L}$ Menthol from in vitro micronucleus assay. Furthermore, the results were compared with the widespread "traditional" ANOVA-like approaches that completely ignore the between-animal variability, focus on p-values and linear trend test only.

\section{MATERIALS AND METHODS}

\section{Data Source Procedure}

$\mathrm{D} / \mathrm{L}$ Menthol was tested in an in vitro micronucleus assay using duplicate mammalian lymphocyte cultures prepared from two mice donors in two independent experiments. To show reproducibility of the results, the two replicate cultures were performed both in the absence and presence of metabolic activation (S9-mix). Mitomycin-C (MMC) at 0.02, 0.03, 0.07, and $0.10 \mu \mathrm{g}$. $/ \mathrm{mL}$ and Cyclophosphamide (CP) at 1.5 and $2.5 \mu \mathrm{g}$. $/ \mathrm{mL}$ were used as the positive controls in the non-activated and activated systems, respectively. Sterile dimethyl sulphoxide (DMS) was added to cultures designated as negative control. The cells were treated at concentrations ranging from 40 to $250 \mu \mathrm{g} . / \mathrm{mL}$, both in the presence and absence of the metabolic activator S-9, until cell harvest. The harvest time was either $4 \mathrm{~h}$ or $24 \mathrm{~h}$ after the initiation of the treatment. The treatment scheme is summarized in Table 1. Finally, around 1000 cells from each culture (around 2,000 per concentration) were analyzed for the presence of micronuclei.

\section{Statistical Analysis}

The frequency of micronuclei as counts was analyzed by pooling the number of MN over culture using a Poisson model. Let $Y_{\mathrm{ij}}$ be the number of micronuclei observed in the $i^{\text {th }}$ culture under concentration $c_{j}, \mathrm{j}=0,1,2, \ldots, \mathrm{m}$. If $\mathrm{Y}_{\mathrm{ij}}$ can be assumed to be distributed independently with

$$
Y_{i j} \sim \operatorname{Poisson}\left(\mu\left(\mathrm{x}_{j}\right)\right)
$$

Then, the log-linear Poisson regression can be applied such that

$$
\mu\left(x_{j}\right)=\exp \left(\alpha+\beta^{T} x_{j}\right)
$$

where $\alpha$ is the $\log$ of mean of the reference group, i.e. vehicle control, $\beta^{\mathrm{T}}$ is a vector of the parameter estimates, and $\mathrm{x}_{\mathrm{j}}$ is a vector of the covariates. In this case, the covariate was the indicator variable for the concentration group. 
Accordingly, to observe any significant differences amongst the dosage sets in assessing the genotoxicity effects, the Dunnet's-type procedure was used to determine if any differed from the vehicle control. trend tests.

Cochran-Armitage (CA) trend test and Williams-type contrast were compared and used as

Table 1. Treatment scheme for the in vitro micronucleus assay on $\mathrm{D} / \mathrm{L}$ Menthol

\begin{tabular}{lclccc}
\hline \multicolumn{5}{c}{ Treatment Condition } \\
\hline Group & $\begin{array}{c}\text { Concentration } \\
\mu g . / m L\end{array}$ & Group & $\begin{array}{c}\text { Concentration } \\
\mu g . / m L\end{array}$ & Group & $\begin{array}{c}\text { Concentration } \\
\mu g . / m L\end{array}$ \\
\hline \hline Conc. 1 & 40 & Conc. 1 & 50 & Conc. 1 & 50 \\
Conc. 2 & 60 & Conc. 2 & 100 & Conc. 2 & 100 \\
Conc. 3 & 80 & Conc. 3 & 150 & Conc. 3 & 125 \\
Conc. 4 & 100 & Conc. 4 & 200 & Conc. 4 & 150 \\
Conc. 5 & 150 & Conc. 5 & 215 & Conc. 5 & 175 \\
Conc. 6 & 175 & Conc. 6 & 225 & Conc. 6 & 200 \\
Conc. 7 & 200 & Conc. 7 & 230 & Conc. 7 & 250 \\
MMC & 0.02 & CP & 1.50 & DMS & 0.00 \\
MMC & 0.04 & CP & 2.50 & MMC & 0.07 \\
DMS & 0.00 & DMS & 0.00 & MMC & 0.10 \\
\hline
\end{tabular}

\section{Statistical Software}

All statistical procedures were implemented in $\mathrm{R}$. The codes used for the analysis were counterchecked by using a user-friendly graphical user interface (GUI) stat4tox which can be downloaded online for free. All analyses were done at 5\% level of significance.

\section{RESULTS AND DISCUSSION}

The in vitro method ensures that the cells were exposed to well-defined concentrations of $\mathrm{D} / \mathrm{L}$ Menthol. The lower one-sided confidence limits for relative risks (RR) for comparisons versus control without an order restriction from the Dunnet-type were given in Tables 2 to 4. Applications of the different concentrations with $\mathrm{D} / \mathrm{L}$ Menthol in the absence and presence of metabolic activation (S-9) in both experiments resulted in frequencies which were similar to and not significantly $(\mathrm{p} \leq 0.05)$ different from those observed in concurrent vehicle controls for all concentrations analyzed. The genotoxic activity of the compound was enhanced in the presence of the metabolic activation system. Clearly, culture grown in the presence of MMC and one level of Cyclophosphamide (CP) showed statistically significant positive responses, i.e. a several-fold increase in micronuclei frequency, as compared to the corresponding controls in all experiments, hence validating the sensitivity of the system to the known mutagenic activity of MMC and $\mathrm{CP}$ under the experimental conditions used. The results presented here point towards a non-genotoxic mechanism behind D/L Menthol. 
Table 2. D/L Menthol, 24 hour treatment in the absence of S-9

\begin{tabular}{llll}
\hline Comparisons & RR & Lower RR & Pvalue \\
\hline \hline Conc. 1 / DMSO & 1.51 & 0.76 & 0.29 \\
Conc. 2 / DMSO & 0.84 & 0.38 & 0.95 \\
Conc. 3 / DMSO & 1.48 & 0.74 & 0.32 \\
Conc. 4 / DMSO & 1.54 & 0.77 & 0.27 \\
Conc. 5 / DMSO & 0.99 & 0.46 & 0.87 \\
Conc. 6 / DMSO & 0.57 & 0.23 & 1.00 \\
MMC1 / DMSO & 3.51 & 1.91 & 0.00 \\
MMC2 / DMSO & 5.66 & 3.16 & 0.00
\end{tabular}

Table 3. D/L Menthol, 4 hour treatment in the absence of S-9

\begin{tabular}{llll}
\hline Comparison & RR & LowerRR & Pvalue \\
\hline \hline Conc. 1 / DMSO & 0.99 & 0.51 & 0.83 \\
Conc. 3 / DMSO & 1.36 & 0.74 & 0.36 \\
Conc. 4 / DMSO & 1.41 & 0.77 & 0.30 \\
Conc. 5 / DMSO & 1.40 & 0.77 & 0.30 \\
Conc. 6 / DMSO & 1.16 & 0.62 & 0.63 \\
MMC1 / DMSO & 4.99 & 3.02 & 0.00 \\
MMC2 / DMSO & 5.50 & 3.35 & 0.00 \\
\hline
\end{tabular}

Table 4. D/L Menthol, 4 hour treatment in the presence of $\mathrm{S}+9$

\begin{tabular}{llll}
\hline Comparisons & $\mathrm{RR}$ & LowerRR & Pvalue \\
\hline \hline Conc. 2 / DMSO & 1.27 & 0.68 & 0.47 \\
Conc. 3 / DMSO & 1.36 & 0.73 & 0.36 \\
Conc. 5 / DMSO & 1.27 & 0.68 & 0.47 \\
Conc. 6 / DMSO & 1.29 & 0.69 & 0.44 \\
CP1 / DMSO & 1.55 & 0.85 & 0.17 \\
CP2 / DMSO & 3.19 & 1.87 & 0.00 \\
\hline
\end{tabular}

The CA test was used to test the trend among the number of micronucleus for in vitro. This test is based on a totally ordered alternative hypothesis to assess the concentration-response relationship. For the three CA tests in the experiment with treatment conditions $24 / \mathrm{S}-9,4 / \mathrm{S}-9$, and $4 / \mathrm{S}+9$, the p-values obtained were $0.8981,0.1362$, and 0.2125 , respectively. The p-values were distinctly larger which favor the null hypothesis of no significant trend among the number of micronuclei over the alternative hypothesis that the number of micronucleus increases with increasing concentration scores. 
In addition to looking at statistical significance of the trend, it is also imperative to assess the biological relevance of the results. For this reason, the Williams-type trend test as recommended by HG was performed which was constructed for both a total order alternative as well as specific comparisons versus control. Tables 5 and 6 give the relative risk estimates and their lower confidence limits for Williams-type contrasts. In this test, the presence of a significant trend in the number of micronuclei with increasing concentration can be concluded if at least one of the lower limit confidence intervals for the relative risks excludes the value of one (Herberich and Horthorn, 2012).

Clearly, no trend can be inferred using the Williams-type contrast because all the $97.5 \%$ lower simultaneous confidence intervals included the value 1 . Moreover, the distance of the lower limits to 1 , ranges from 0.01 to 0.44 , was small that toxicologically insignificant is a very likely conclusion. This is, in comparison to CA test, the advantage of Williams-type test wherein the lower limit of the relative risk can be used to interpret the effect size in terms of biological relevance. Furthermore, this test provides more information regarding the dose-response shape than a simple p-value for a global test of trend (Herberich and Hothorn 2012).

Table 5. Williams-type contrasts for relative risks on the number of MN (24h/-S9)

\begin{tabular}{lll}
\hline Comparison & RR & LowerRR \\
\hline C1: Vehicle vs. Concentration 6 & 0.56 & 0.26 \\
C2: Vehicle vs. Concentrations 6 and 5 & 0.75 & 0.41 \\
C3: Vehicle vs. Concentrations 6, 5, and 4 & 0.96 & 0.55 \\
C4: Vehicle vs. Concentrations 6, 5, 4, and 3 & 1.06 & 0.63 \\
C5: Vehicle vs. Concentrations 6, 5, 4, 3, and 2 & 1.01 & 0.61 \\
C6: Vehicle vs. Concentrations 6, 5, 4, 3, 2, and 1 & 1.09 & 0.66 \\
\hline
\end{tabular}

Table 6. Williams-type contrasts for relative risks on the number of MN (4h/+S9)

\begin{tabular}{lll}
\hline Comparison & RR & LowerRR \\
\hline C1: Vehicle vs. Concentration 6 & 1.29 & 0.75 \\
C2: Vehicle vs. Concentrations 6 and 5 & 1.28 & 0.80 \\
C3: Vehicle vs. Concentrations 6, 5, and 3 & 1.31 & 0.83 \\
C4: Vehicle vs. Concentrations 6, 5, 3, and 2 & 1.30 & 0.83 \\
\hline
\end{tabular}

Collectively, findings revealed that $\mathrm{D} / \mathrm{L}$ Menthol did not induce a clear dose-dependent increase in the micronuclei occurrence. Though the culture was the randomized experimental unit, it would be more appropriate if the between-cultures variability was taken into account. However, because of the commonly used two cultures only, the estimation of a related dispersion parameter for proportion/count is rather unstable. This means if more cultures are available, a model with extra-variation like quasi-Poisson model is recommended. However due to the issue of sample size, a Poisson model was fitted for the pooled-over culture data, i.e. pooling all the number of micronuclei over a culture.

\section{Acknowledgments}

This paper was extracted from the author's Master Thesis entitled Statistical Evaluation of In vivo and In vitro Micronucleus Assays in Toxicology which was undertaken with full support under the Belgian government through its Vlaamse InterUniversitaire Raad (VLIR) Scholarship

Vol. 1 No. 2 (December 2016) ISSN: 2507-9638 DOI: 10.22137/ijst.2016.v1n2.01 
Program. The author greatly appreciates Prof. Dr. Helena Geys of Hasselt University, Diepenbeek, Belgium for providing the author the data and for her valuable guidance and directives through the learning process of this project.

\section{REFERENCES}

Bretz, F. and L.A. Hothorn. 2002. "Detecting Dose-response Using Contrasts: Asymptotic Power and Sample Size Determination for Poisson Data." Stat Med. 21 (22): 3325-3335.

Herberich, E. and L.A. Hothorn. 2012. "Statistical Evaluation of Mortality in Long-term Carcinogenicity Bioassays Using a Williams-type Procedure." Regulatory Toxicology and Pharmacology 64: 26-34.

Hothorn, L.A. and D. Gerhard. 2009. "Statistical Evaluation of the In Vivo Micronucleus Assay." Arch Toxicol 83: 625-634.

Hothorn, L.A. and D. Gerhard. 2008. Statistical Evaluation of the In Vitro Micronucleus Assay. Reports of the Institute of Biostatistics No. 09/2008.

OECD 487. 2009. OECD Guideline for the Testing of Chemicals: Draft Proposal for a New Guideline: In Vitro Mammalian Cell Micronucleus Test (Mnvit). 